\title{
Perspectivas plurales en investigación cualitativa: propuestas metodológicas en estudios de gestión
}

\begin{abstract}
Plural perspectives in qualitative investigation: methodological proposals in management studies
\end{abstract}

Perspectivas plurais em pesquisa qualitativa: propostas metodológicas em estudos de gestão

\author{
Camilo Páez Quevedo \\ Universidad Andina Simón Bolívar, Sede Ecuador \\ cpaez80@gmail.com \\ https://orcid.org/0000-0003-3410-3633
}

DOI: https://doi.org/10.32719/25506641.2020.7.2

Recibido: 1 de septiembre de 2019 • Revisado: 21 de octubre de 2019

Aceptado: 15 de noviembre de 2019

Artículo de investigación

Licencia Creative Commons

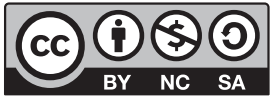




\section{Camilo Páez Quevedo}

\section{Resumen}

En el campo de la administración, el horizonte metodológico se enfrenta a la naturaleza de un objeto de estudio signado por lo histórico, contingente y complejo, por lo que es necesario transitar hacia un ethos metodológico alternativo, de formas de razonamiento y lógicas distintas a las implementadas en la investigación positivista. Desde el planteamiento citado, este trabajo presenta una perspectiva metodológica pospositivista en la cual confluyen el enfoque cualitativo y los abordajes crítico-interpretativos, con el fin de recuperar la mediación filosófica para la investigación en gestión. La dinamización del campo metodológico que proponemos prevé la estructuración de tres espacios lógicos de investigación, a saber: a) un enfoque metodológico crítico-interpretativo; b) una estrategia de intelección; y c) unas técnicas investigativas. Estos espacios lógicos se imbrican y asientan en una visión filosófica y epistémica; en el presente caso, la teoría crítica de la Escuela de Fráncfort. La investigación se ilustra a partir de un estudio sobre la formación en gestión del talento humano en Ecuador, con el propósito de realizar una aproximación a las perspectivas plurales de los estudios organizacionales.

Palabras clave: Filosofía, teoría crítica, estudios organizacionales, metodología cualitativa, reducción sociológica, discurso.

JEL: B5 Enfoques heterodoxos actuales.

\section{Abstract}

In the field of administration, the methodological horizon faces the nature of a study object marked by the historical, contingent and complex, thus it is necessary to move towards an alternative methodological ethos, different from those implemented in positivist research in reasoning and logical forms. From the aforementioned approach, this paper presents a post-positivist methodological perspective in which the qualitative approach converges with critical interpretative approaches and recovers the philosophical mediation for management research. The dynamization of the methodological field that we propose foresees the structuring of three logical research spaces, i) a critical-interpretative methodological approach; ii) an intellection strategy; iii) research techniques. These logical spaces are embedded and set in a philosophical and epistemic vision, in our case the Critical Theory of the Frankfurt School. The research is illustrated by a study on human talent management training in Ecuador and is aligned with the purpose of approaching plural perspectives in Organizational Studies.

Keywords: Philosophy, critical theory, Organizational studies, qualitative methodology, sociological reduction, discourse.

JEL: B5 Current heterodox approaches.

\section{Resumo}

No campo de administração, o horizonte metodológico se enfrenta à natureza de um objeto de estudo marcado pelo histórico, contingente e complexo, razão pela qual é necessário transitar para um ethos metodológico alternativo, de formas de raciocínio e lógicas 
distintas às que foram implementadas na pesquisa positivista. Desde a posição citada, este trabalho apresenta uma perspectiva metodológica pós-positivista na qual confluem o enfoque qualitativo e abordagens crítico-interpretativas e que recupera a mediação filosófica para a pesquisa em gestão. A dinamização do campo metodológico que propomos prevê a estruturação de três espaços lógicos de pesquisa, a saber: i) enfoque metodológico crítico-interpretativo; ii) estratégia de intelecção; e, iii) técnicas de pesquisa. Estes espaços lógicos se imbricam e se assentam em uma visão filosófica e epistêmica, em nosso caso, a Teoria Crítica da Escola de Frankfurt. A pesquisa é ilustrada a partir de um estudo sobre a formação em gestão do talento humano no Equador e se alinha ao propósito de nos aproximarmos a perspectivas plurais nos Estudos Organizacionais.

Palavras-chave: Filosofia, teoria crítica, Estudos organizacionais, metodologia qualitativa, redução sociológica, discurso.

JEL: B5 Enfoques heterodoxos atuais.

\section{Introducción}

E 1 objetivo planteado en la investigación de origen para la elaboración de este artículo estaba dirigido al alcance de la teoría crítica y de los enfoques metodológicos pospositivistas como capaces de problematizar el devenir en la gestión del talento humano y la capacidad de constituirse como un ethos investigativo, desde el locus no funcionalista. Alrededor de este horizonte se organizaron los cuestionamientos epistemológicos y teórico-metodológicos, los cuales definen también los objetivos de investigación: en primer lugar, la necesidad de lograr una aproximación a las centralidades semánticas y los elementos de tensión epistemológica inherentes a la disciplina -objeto de nuestro estudio-; y segundo, entender cómo las centralidades semánticas y los elementos de tensión podrían conformar un ethos investigativo.

En cuanto el primer objetivo, la decisión investigativa consistió en plantear una pesquisa de indicadores bibliométricos y conceptos centrales que conformaban la producción investigativa. Para el desarrollo del segundo objetivo nos aprestamos al análisis de las conformaciones míticas en el quehacer investigativo.

La investigación empírica fue desarrollada a lo largo del año 2017 y tuvo como fuente secundaria el conjunto de tesis del programa de posgrado en Desarrollo del Talento Humano de una institución de educación superior en 
Quito, Ecuador. Mediante el estudio planteado, se pretendió encontrar las características básicas, materializadas en la narratividad de las tesis de maestría de una formación pensada desde Ecuador y desde una perspectiva latinoamericana. Estos elementos dieron sustento al interés de reflexionar acerca de, por un lado, el potencial teórico-conceptual y, por otro, el potencial metodológico y heurístico que la teoría crítica y los enfoques pospositivistas de investigación podrían tener, en la línea de superar los modelos explicativos de investigación sustentados en el pensamiento positivista.

\section{Problematización}

En la gestión del talento humano, el cuerpo de conocimientos que trata los fenómenos alrededor de la persona-trabajo-organización ha tenido una formación centrada principalmente en la dimensión práctica-instrumental, en la que ha prevalecido el dominio de lo técnico por sobre lo teórico-conceptual. Esta situación ha conllevado diversas implicaciones; la principal -y aquella que penetra en las demás- es la ausencia de una reflexión teórico-conceptual y filosófica que coadyuve como marco orientador del programa científico de la disciplina.

Se entiende que para la problematización de esta dimensión del pensamiento organizacional y administrativo puede $-\mathrm{y}$ debe- haber múltiples aproximaciones: desde el orden de lo académico, lo profesional y/o lo investigativo; este último, un momento crítico del proceso del conocimiento, en tanto es el momento de producción de la ciencia. Este momento científico se materializa en la producción investigativa local, la cual refleja el estado de arte de la disciplina y se expresa a través de varias tipologías documentales, como por ejemplo la tesis de maestría.

La forma positivista de abordar el objeto de estudio ha sido el referente hegemónico en las ciencias sociales, como resultado de una extrapolación de las ciencias naturales a las ciencias humanas. Este método de captación de la realidad, que se basaba en la aprehensión inmediata de epifenómenos, deja de lado importantes problemáticas de la subjetividad humana y organizacional. En este sentido, a partir del desarrollo de algunos enfoques teóricos tales como "la filosofía posanalítica de Wittgenstein, el posestructuralismo 
de Barthes, la deconstructiva doble lectura de Derrida, el neopragmatismo de Rorty, la aproximación genealógica de Foucault y el psicoanálisis de Lacan" (Torfing 2004, 34), las ciencias sociales dirigen su atención a fenómenos a partir de la mediación del lenguaje, para dar paso al reconocimiento de importantes narrativas, discursos y elementos de tensión en la dimensión organizacional, y particularmente en la relación laboral.

Considerando dichos antecedentes, este artículo se dedica a presentar las decisiones metodológicas emprendidas para el objetivo planteado, lo cual implica entender los distintos problemas a la luz de los fundamentos narrativos que tienen los fenómenos sociales y humanos.

En este sentido, desarrollamos un modelo de análisis considerando las pluralidades epistemológicas del campo científico de la administración y de los estudios organizacionales, y concentrándonos en la estructuración de un enfoque metodológico que contemple la mediación filosófica. Consecuentemente, nuestra propuesta plantea la necesidad de considerar varios espacios metodológicos que se articulen entre sí y que fortalezcan el proceso investigativo.

\section{Lineamientos epistemológicos y teóricos sobre lo metodológico}

El eje de los lineamientos metodológicos del estudio realizado se caracteriza por la presencia de algunos niveles investigativos o espacios lógicos, tales como: a) una posición ontoepistémica como marco orientador del ejercicio investigativo y la reflexión científica; b) un enfoque metodológico crítico e interpretativo de investigación, que sirva de marco orientador del rumbo epistémico del estudio; c) una estrategia de intelección que nos permita principios vertebradores para el análisis, coherente con el enfoque crítico y pospositivista; y d) técnicas investigativas para el procesamiento del referente y el material empírico.

Los niveles investigativos y aspectos metodológicos señalados guardan entre sí una relación lógica, deben ser complementarios. Cada espacio metodológico tiene una función en el procesamiento del referente empírico, y se diferencia de los demás en cuanto a la cercanía que tiene con los fenómenos 
acerca de los cuales versa la investigación. Así, por ejemplo, la posición ontoepistémica proporciona abordajes, lógicas y posibilidades de intelección, pero no tiene la capacidad de tratar aspectos específicos de la pesquisa investigativa, ya que este lugar metodológico lo ocupan lo que Merton (1992) denominó teorías de alcance intermedio. En la figura 1 se ilustra cada uno de los niveles investigativos o espacios lógicos citados.

\section{Figura 1}

\section{Propuesta metodológica de investigación pospositivista}

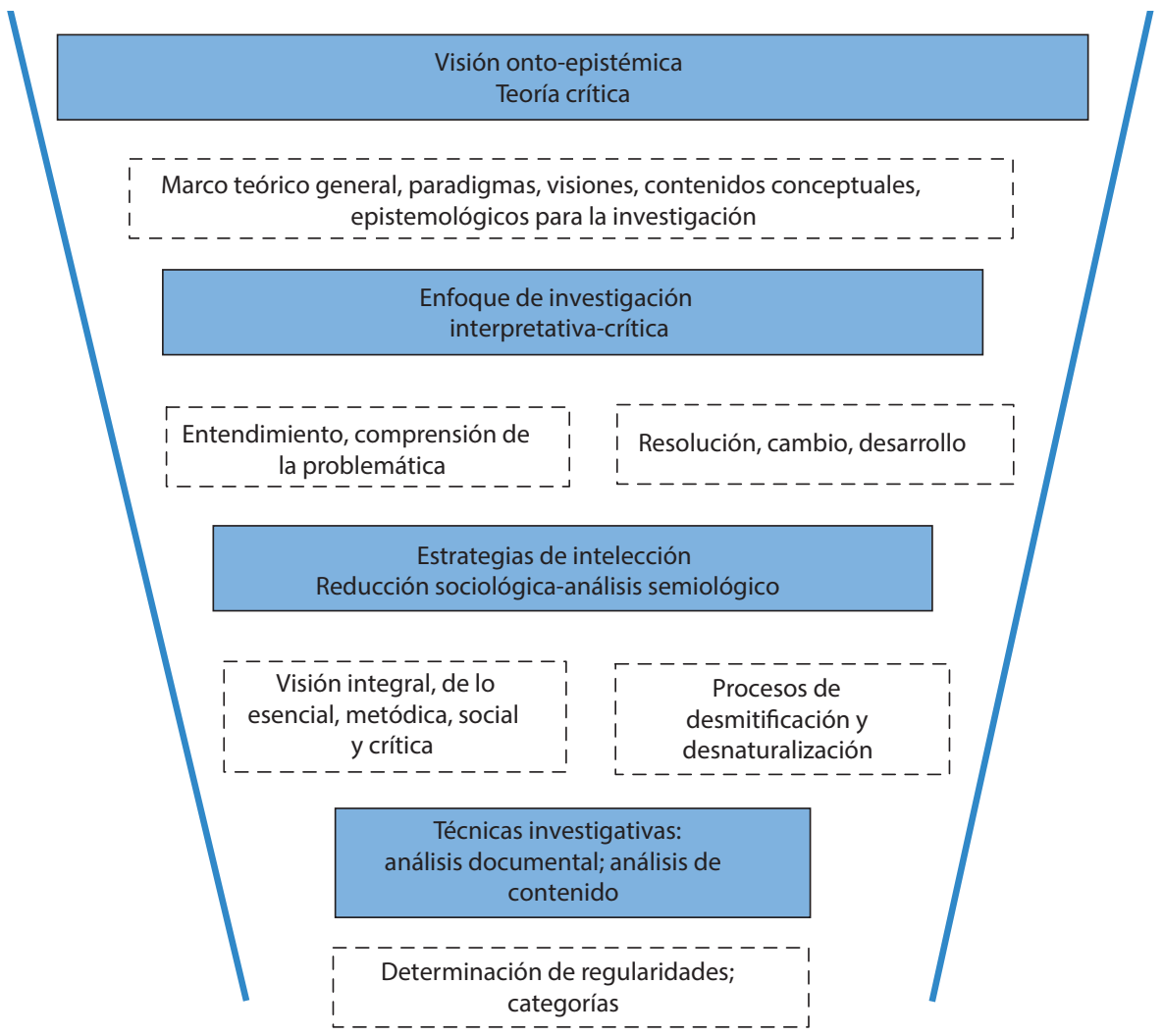

Fuente y elaboración propia. 
Creemos que proponer un horizonte metodológico pospositivista es consistente, en primer lugar, con las necesidades disciplinares del campo de estudio en el que nos centramos; en segundo lugar, con las necesidades de problematización y complejización que tienen muchos fenómenos en este campo; y en tercero, con el enfoque filosófico -ontoepistémico- asumido, esto es, desde la teoría crítica de la Escuela de Fráncfort.

Planteamos que el primer nivel investigativo o espacio lógico sea la posición ontoepistémica, que consiste en el posicionamiento, los paradigmas más amplios y profundos de los cuales parte el investigador. Este nivel implica un alto grado de abstracción, en tanto condensa elaboraciones filosóficas, cosmovisivas, epistemológicas y teóricas, las cuales, en conjunto, permiten al investigador un amplio nivel de abarcabilidad y de intelegibilidad frente a los fenómenos. Una investigación que se proponga la interpelación de problemáticas debe partir necesariamente de un posicionamiento explícito que permita un marco orientador articulable con los otros niveles investigativos. En el caso de la presente investigación, esa posición ontoepistémica está dada por la teoría crítica de la Escuela de Fráncfort.

El segundo nivel investigativo está dado por la asunción de un enfoque metodológico que posibilite la estructuración lógica de la estrategia investigativa y que permita la articulación de las herramientas de análisis del referente empírico. En esta investigación partimos de un enfoque crítico-interpretativo.

La investigación interpretativa "se encuentra en las tradiciones filosóficas de la filosofía analítica del lenguaje, la hermenéutica y la fenomenología" (White 2013, 87). Si bien es cierto que se trata de tradiciones intelectuales diferentes, "comparten un interés por entender la acción en vez de explicar la conducta" (White 2013, 87). Los abordajes de esta corriente de pensamiento aúnan distintas perspectivas, que van desde la estructura lógica del lenguaje ordinario y del lenguaje científico - la filosofía analítica-, pasan por las distintas teorías de interpretación de disciplinas como la literatura, el arte y la religión -la hermenéutica-, y también constituyen el análisis de las estructuras de la experiencia -la fenomenología-. El objetivo de la investigación interpretativa es la comprensión, "el entendimiento de las creencias, significados, sentimientos y actitudes de los actores en las situaciones sociales" (White 2013,89), así como el entendimiento mutuo con el investigador. $\mathrm{Su}$ 
lógica de investigación se basa en que el entendimiento surge de una relación recíproca y circular entre los actores del proceso de conocimiento, y entre las premisas de la situación investigada.

La investigación crítica, por su parte, proviene de "la teoría crítica contemporánea, la crítica neomarxista de la ideología que hace la Escuela de Fráncfort y el psicoanálisis freudiano" (White 2013, 96). Asimismo, estas tradiciones "comparten una preocupación común por la reflexión y la autorreflexión como formas de ayudar a que las personas superen limitaciones" (White 2013, 96). En este sentido, el objetivo de este tipo de investigación consiste en la superación de situaciones opresivas en la búsqueda de libertad y desarrollo. Su lógica investigativa es la autorreflexión sobre la base de la relación propia con el mundo, con un componente de movilización hacia el enfrentamiento de la situación investigada o vivida. A diferencia de la investigación interpretativa, la crítica puede o no tener una relación estrecha entre el investigador y los actores objeto de investigación.

Una de las consideraciones especiales que hacemos en cuanto al componente metodológico del estudio es la necesidad de asumir un enfoque ecléctico, planteado desde la reflexión de la posición ontoepistémica asumida; en nuestro caso, la teoría crítica de la Escuela de Fráncfort. Es decir, para la construcción del abordaje metodológico, consideramos la posibilidad de hacer préstamos analíticos que desde otras disciplinas nos permitan desarrollar y desencadenar las reflexiones acerca de nuestro referente empírico.

En este sentido, el tercer nivel metodológico está dado por la estrategia de intelección. En esta investigación se han determinado, en este nivel, dos formas comprensivas provenientes de las disciplina sociológica y de la disciplina semiológica, respectivamente: a saber, la reducción sociológica propuesta por el sociólogo brasileño Alberto Guerreiro Ramos (1945-1982), y el análisis semiológico para la desmitificación, propuesto por el semiólogo francés Roland Barthes (1915-1980). El papel de la estrategia de intelección es coadyuvar a una articulación entre todos los niveles metodológicos, para cumplir con los objetivos y la dirección del estudio.

La articulación metodológica planteada, particularmente la estrategia de intelección, procura que en un nivel cada vez más cercano al objeto de estudio se faciliten la problematización, la complejización del referente empírico y el cumplimiento de los objetivos investigativos. En otras palabras, cumple 
el papel metodológico de mediar entre el enfoque de investigación y las técnicas investigativas adoptadas.

El valor de la propuesta planteada está justamente en el encuentro de estos dos enfoques, apoyados también en la defensa de perspectivas plurales en los estudios organizacionales y en el planteamiento pluriparadigmático que caracteriza al debate reciente en el campo (Paes de Paula 2001; 2015).

El cuarto y último nivel metodológico corresponde a las técnicas investigativas que se han utilizado en el estudio. A través de ellas obtenemos la información del referente empírico, en este caso las tesis de maestría en el área de talento humano. Este nivel es importante en cuanto a partir de las técnicas se extraen los hallazgos, las características y los elementos constitutivos del estudio, para luego, a partir de los parámetros del enfoque investigativo y las estrategias de intelección, evidenciar y comprender las regularidades y redes de significancia que se proponen en la investigación. En el caso de nuestra investigación se utilizaron las técnicas de análisis documental y análisis de contenido.

Nos interesa detenernos en especial en el nivel de las estrategias de intelección de nuestro modelo, mediante su profundización.

\section{La reducción sociológica}

Los orígenes de la reducción sociológica se pueden encontrar en la fenomenología de Edmund Husserl (1859-1939) y de Martin Heidegger (18891976). El movimiento fenomenológico surge como un intento de hacer dialogar a la filosofía con la ciencia, desde una crítica de la ciencia moderna y del fenómeno dicotómico en el cual devino, caracterizada por el objetivismo y el subjetivismo, lo que sería responsable por "la crisis de sentido" (Hottois 1999, 257).

En síntesis, se puede decir que el programa de la fenomenología fue el retorno de la ciencia a las cosas mismas y a la vivencia de la conciencia. Guerreiro Ramos lo plantea de la siguiente manera: "Husserl, en busca de un conocimiento de las esencias, procura conducir al sujeto hacia la experiencia trascendental en la que solamente puede ocurrir el enfrentamiento del yo puro con el objeto puro" $(1959,114)$. 
Heidegger plantea un principio que será clave en la propuesta de la reducción sociológica que hace Guerreiro Ramos: cada objeto en el mundo participa de una estructura referencial que le da sentido. El sujeto cognoscente, que forma parte de este contexto, de esta estructura referencial, adquiere una comprensión acerca de los objetos que no es aún una comprensión teórica sino tangencial, empírica.

Uno de los aspectos importantes en la propuesta de la reducción sociológica es el sentido inherente a los objetos, de acuerdo a su intencionalidad en la estructura referencial. Otro aspecto esencial que Guerreiro Ramos explicita es la abstracción que hace de la posición idealista tanto de Husserl como de Heidegger; esto, sin embargo, impide retomar la "actitud metodológica" que plantean estos autores.

Guerreiro Ramos define a la reducción sociológica como "una actitud metódica que tiene por finalidad descubrir los presupuestos referenciales, de naturaleza histórica, de los objetos y hechos de la realidad social" $(1959,96)$. Un aspecto complementario pero cardinal en este concepto de la realidad social es que esta surge no solamente como un proceso cognoscitivo del investigador, sino motivada por la necesidad social de una comunidad concreta.

Las características de la reducción sociológica que plantea Guerreiro Ramos nos permite importantes aproximaciones a nuestro objeto de estudio, aproximaciones que intentarán su problematización. En su libro La reducción sociológica: introducción al estudio de la razón sociológica, el autor realiza dos ilustraciones de reducción sociológica: de un concepto y de una técnica de investigación social. En el primero de ellos, el autor argumenta la poca funcionalidad que tiene el concepto de "control social" para la realidad brasileña, mientras que para el caso de la realidad norteamericana es altamente necesaria. En la reducción sociológica de la técnica de investigación, desarrolla el hecho de que los resultados obtenidos por la técnica mencionada han de ser interpretados a partir de escalas de consumo alimenticio ajustadas a la realidad de la sociedad brasileña, y no basándose en escalas que pertenezcan a otras realidades.

Como se manifestaba, la estrategia de intelección que hemos explicado -la reducción sociológica- cumple el papel metodológico de mediar entre lo que hemos denominado el enfoque de investigación y las técnicas investigativas adoptadas. La estrategia de intelección o forma de comprensión que 
implementamos nos permite un procesamiento más cercano del objeto de estudio. En el caso concreto de la reducción sociológica, utilizamos este conjunto de principios como matriz de intelección para el análisis de contenido (apoyado en el software NVivo), el cual nos ha permitido la identificación de los conceptos más citados y las frases asociadas a ellos. Hemos analizado estas preposiciones mediante un proceso de identificación del contenido de las citas, realizado a través de los principios de la reducción sociológica. Sin la utilización de este recurso metodológico, el análisis de contenido carecería de una orientación más limitada al contexto y de las premisas analíticas que permitan captar lo esencial del material empírico -en el caso de esta investigación, los árboles de conceptos obtenidos del sistema NVivo--

\section{El análisis semiológico para la desmitificación de Roland Barthes y el mito organizacional}

Otra de las mediaciones utilizadas en la presente investigación lo constituyen el análisis y la interpretación semiológica propuestos por Barthes, quien sistematiza los desarrollos de fenómenos del lenguaje de autores como Ferdinand de Saussure. En su clásica obra Mitologías, Barthes se propone una "crítica ideológica dirigida al lenguaje de la llamada cultura de masas; por otra parte, plantea un primer desmontaje semiológico de ese lenguaje" $(2008,11)$.

Se optó por esta perspectiva de análisis semiológico del mito que, en primer lugar, permite evidenciar la intencionalidad ideológica presente en la ciencia, es decir, la exposición de ideas, hechos y fenómenos como algo natural, vaciándolos de su contenido eminentemente histórico. En segundo lugar, dado que el estudio asume el discurso como la herramienta a partir de la cual se da inteligibilidad a los distintos fenómenos, vemos en el mito una forma de lenguaje presente en la discursividad de los ámbitos organizacionales. ${ }^{1}$

En la formación mítica existe una ambivalencia entre sentido y forma como expresiones del significante. Como sentido, el significante evoca una lectura: se lo capta, se lo percibe. Como forma, es captado únicamente a tra-

1. Aclaramos que el análisis de las conformaciones míticas es solo una de las ramas de la disciplina semiológica. 
vés de la medición psíquica y cultural. Cuando el sentido deviene en forma, se empobrece, se debilita; opera -según manifiesta Barthes- una regresión anormal del sentido a la forma. La pobreza actual de la forma requiere una significación que la reemplace, así como la forma necesita alimentarse del contenido, de la historia inherente al sentido. En ese sentido, Barthes plantea que "lo que define el mito es este interesante juego de escondidas entre el sentido y la forma" $(2008,210)$.

Analizados los elementos de sentido y forma, vamos al significado, es decir, al concepto: este es de naturaleza absolutamente histórica e intencional. Es a través del concepto que se implanta una historia nueva, la conformación mítica en sí. La dinámica regresiva del sentido en forma sucede para recibir un concepto nuevo. El concepto deforma, pero no elimina el sentido, sino que lo aliena. Un aspecto señalado por Barthes es que un significado puede tener innumerables significantes.

La significación, por su parte, es la unión de los dos primeros; es la concreción del mito en sí. En la significación se cierra el proceso mítico, que inicia con la presencia de un significante en su perspectiva de sentido y de forma. Este significante se vacía, se empobrece por el contexto en el cual se genera; entonces, la presencia del significado como concepto se manifiesta en toda su precisión y se opera una suerte de despojo de todos los elementos históricos inherentes. De esta manera se presenta la significación, materialización discursiva del mito.

Finalmente, toda formación mítica despliega una estrategia que le es constitutiva y necesaria: la naturalización. Ante la eventualidad de que el consumidor de mitos perciba el concepto y su significación -sea porque realiza una lectura inocente o una reflexiva-, el mito elabora un segundo sistema semiológico que le impida liquidar al concepto; por tanto, lo naturaliza. "Estamos en el principio mismo del mito: él transforma la historia en naturaleza" (Barthes 2008, 222).

Resumimos aquí algunos motivos que hacen del análisis semiológico una herramienta de intelección útil y de alto potencial heurístico para el análisis organizacional de la gestión de talento humano en las organizaciones, así como de la investigación realizada: 
1. El fenómeno organizacional, en particular el que se da en el ámbito del talento humano, constituye dinámicas sociales altamente susceptibles de analizarse desde la narratividad y el discurso.

2. En el mundo organizacional hay una gran carga de sugestividad, de expresividad de los actores, lo cual contiene información, valoraciones y subjetividades que deben analizarse.

3. Esta expresividad conforma un material que en el ámbito organizacional, por determinadas motivaciones, conforma un lenguaje mítico que conlleva mensajes que fundamentan las prácticas organizacionales.

4. Las formas del lenguaje mítico son diversas, no solamente orales. Aparecen en toda forma de discursividad: imágenes, símbolos, rituales y todo tipo de tipología escrita-como políticas, manuales, procesos, revistas...-.

5. El significante lingüístico está representado en el ámbito organizacional por -valga la redundancia- el significado lingüístico presente, por ejemplo, en el Diccionario de la Lengua de la Real Academia Española. Dada la amplitud del significante, tanto lingüístico como mítico, este tiene muchas formas de representación.

6. Dada la complejidad y diversidad de las formas discursivas en el ámbito organizacional, la estructuración mítica adquiere algunas particularidades. Partiendo del modelo de Barthes, el significante mítico es diverso, adquiere múltiples formas, tales como relatos, disposiciones, opiniones profesionales, experiencias, testimonios, etc.

7. El concepto mítico - significado- se representa a través de la opinión científica y la literatura académica e institucional.

8. La significación mítica-signo- es la postulación del mito en sí. Se representa a través de postulados, principios, relatos, declaraciones, etc., los cuales en su expresión están atravesados por características de jerarquía, poder, dominio y exclusión.

\section{Metodología}

Expuestos los lineamientos teóricos del enfoque metodológico, nos corresponde presentar los aspectos esenciales de la implementación de la investigación para cumplir con los objetivos planteados. Para el primer objeti- 
vo de investigación, nos propusimos la definición de categorías de análisis, las cuales fueron divididas en formales y de contenido. Las formales contemplaron el área temática, el tipo de organización, el género de los autores, la conformidad con el formato de presentación y el alineamiento a las líneas de investigación del programa. En relación a las categorías de contenido, se verificó la consistencia y coherencia en el planteamiento del problema, en la definición de un diseño metodológico, en la indicación de técnicas utilizadas y en las reflexiones presentadas.

Posteriormente se realizaron dos procedimientos, apoyados por un sistema de análisis cualitativo denominado NVivo; para ello definimos como la unidad de análisis a la palabra. El procedimiento realizado consistió en la determinación de conceptos de mayor frecuencia de designación; esto nos permitió posteriormente lo que hemos denominado la determinación de centralidades semánticas, también a partir del recurso denominado árbol de palabras, del mismo sistema de análisis cualitativo.

Para el segundo objetivo de investigación, se buscó identificar las construcciones míticas en los trabajos analizados, lo que se realizó determinando tres conceptos centrales surgidos del análisis de contenido. De esta forma, apoyados en las premisas del modelo de construcción mítica de Barthes, se identificaron las regularidades discursivas que conforman una estructura mítica.

\section{Tejiendo conclusiones}

El análisis cualitativo desde las narrativas, las construcciones semánticas y las discursividades posibilita otras formas de captación de la realidad social, así como nuevas críticas, reflexividades y comprensiones sobre un objeto de estudio. Además, plantea que existe un ethos investigativo -además de académico y profesional- que se conforma a partir del discurso mítico y se convierte en un recurso ideológico para el mantenimiento de una ciencia -y sociedad- mítica.

La investigación explicativa en talento humano y áreas organizacionales, consecuente con sus fundamentos filosóficos de orientación positivista, piensa la realidad organizacional como un fenómeno a partir del cual se pueden 
extraer conclusiones inequívocas y universales. Esta posición esencialista simplifica la realidad y desconoce las singularidades, las subjetividades propias de un objeto de estudio de naturaleza histórica, esencialmente contingente e impregnado de subjetividad.

En la búsqueda de predecir comportamientos tanto individuales como grupales, dejamos de lado la riqueza que podría significar una comprensión integral de los fenómenos. En su lugar, perseguimos la manipulación de las variables bajo la lógica de causa y efecto, para la consecución de objetivos que no están relacionados con la superación y resolución de contradicciones, sino simplemente con la comprobación per se de hipótesis. Desde esta perspectiva, el programa científico es de administrar conocimiento técnico, y no desarrollar conocimiento teórico que lo fundamente y que permita la superación de problemas sociales en el área.

Con su lógica y modo de razonamiento, la investigación explicativa está condicionada por un examen lineal de los problemas, ya sea a partir de análisis deductivos o inductivos; se deja de lado una lógica de interpretación y reflexión, necesaria para el tipo de problemas de naturaleza eminentemente sociopsicológica. Se llega así a una formación -sea de un científico, académico o profesional- acrítica, irreflexiva, comprometida solo con responder a determinadas necesidades de las organizaciones, pero sin la capacidad de crear necesidades en la sociedad en la que se inserta.

Al no plantear métodos que impliquen al investigador con el sujeto estudiado, que permitan que este se involucre y empodere como conocedor consciente de su realidad, reducimos la posibilidad de comunicar los aspectos investigados, de construir una relación humana con el sujeto investigado, con su problemática y con la sociedad. Otras contradicciones limitan su capacidad de profundización de los fenómenos al desplegar herramientas principalmente cuantitativas en la recopilación de información del objeto de estudio, ya que constriñen los fenómenos humanos y organizacionales, y queda invisible la trama de significaciones que se despliegan en toda su compleja expresión.

La propuesta metodológica de este artículo vincula el análisis semiológico desde la construcción mítica con la reducción sociológica de Guerreiro Ramos, como una de las vías de problematización del referente empírico. 
Esta mirada tiene importantes antecedentes en corrientes filosóficas y de pensamiento pospositivistas que pueden contribuir al análisis y la superación de los problemas de la disciplina, desde un enfoque contextualizado localmente. La parte práctica se relaciona con la identificación del tipo de problemas que abordan las investigaciones, y con el correlato conceptual que emerge a partir de este abordaje. Hemos pretendido problematizar en primer lugar cómo la génesis investigativa se asienta principalmente en las necesidades empresariales dentro del contexto de un tipo particular de sociedad -la industrial- para, con ello, evidenciar la naturalización de determinadas visiones y prácticas, las cuales determinan a su vez singulares comportamientos investigativos (en consecuencia, profesionales y académicos). A partir de esta naturalización se gesta un proceso de conformación de mitos que constituye la base cultural de la disciplina.

\section{Referencias}

Barthes, Roland. 2008. Mitologías. Buenos Aires: Siglo XXI.

Guerreiro Ramos, Alberto. 1959. La reducción sociológica: introducción al estudio de la razón sociológica. Ciudad de México: Universidad Nacional Autónoma de México / Instituto de Investigaciones Jurídicas Sociales.

Hottois, Gilbert. 1999. Historia de la filosofía del Renacimiento a la posmodernidad. Madrid: Cátedra.

Merton, Robert K. 1992. Teoría y estructuras sociales. Ciudad de México: Fondo de Cultura Económica.

Paes de Paula, Ana Paula. 2015. Repensando os estudos organizacionais: por uma nova teoria do conhecimento. Río de Janeiro: FGV Editora.

Torfing, Jacob. 2004. "Un repaso al análisis de discurso". En Debates políticos contemporáneos: en los márgenes de la modernidad, coordinado por Rosa Buenfil Burgos, 31-53. Ciudad de México: Plaza y Valdés.

White, Jay. 2013. Tomar en serio el lenguaje: los fundamentos narrativos de la investigación en administración pública. Ciudad de México: Fondo de Cultura Económica. 